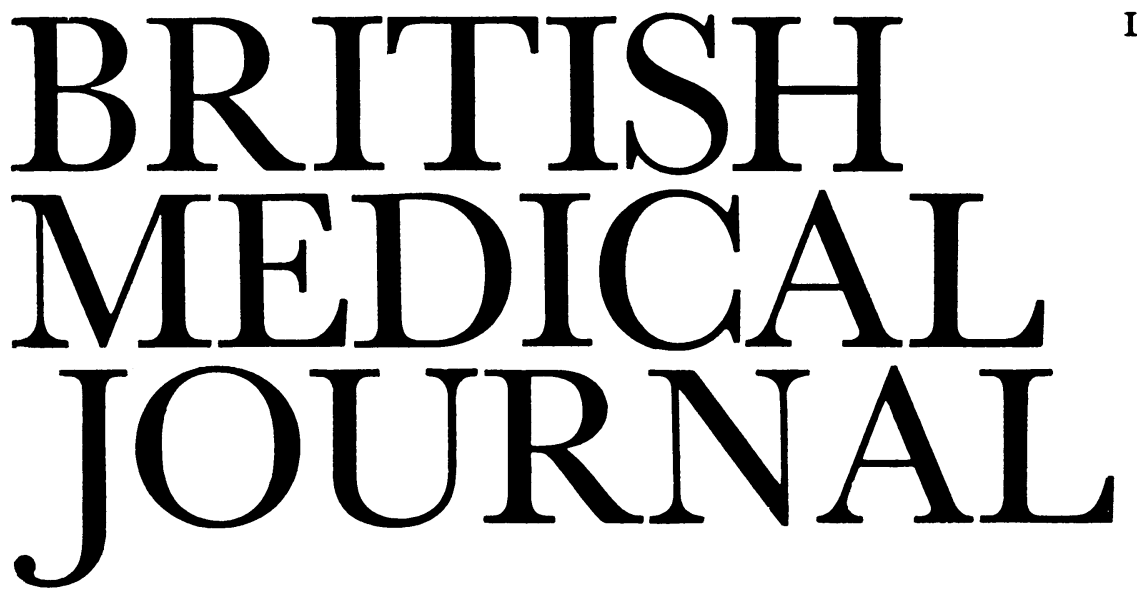

LONDON, SATURDAY 11 FEBRUARY 1984

\title{
Hypercholesterolaemia and coronary heart disease: an answer
}

At long last we have clear evidence that reducing very high plasma concentrations of cholesterol and low density lipoprotein (LDL) cholesterol lowers the incidence of coronary heart disease. The United States Lipid Research Clinics trial has shown that reduction of these lipids was associated with a significant reduction in the risk of coronary heart disease (deaths and non-fatal infarcts) by $19 \%$ after seven years. ${ }^{1}$ There were also decreases in angina $(-20 \%)$, the development of electrocardiographic signs on exercise testing $(-24 \%)$, and the rate of treatment by coronary bypass surgery $(-21 \%)$.

The patients treated were, however, a clearly defined group at high risk. They were 3806 men in good health and free from any symptoms of coronary heart disease aged between 35 and 59 (mean 48) in 12 North American clinical centres selected from those in the top $5 \%$ of the frequency distribution of cholesterol and categorised as having type II hyperlipoproteinaemia. $^{2}$ They were, therefore, well motivated to cooperate for the seven years of the trial. The mean baseline concentration of plasma cholesterol was $7.6 \mathrm{mmol} / 1$ (292 $\mathrm{mg} / 100 \mathrm{ml}$ ) and of LDL cholesterol $5.6 \mathrm{mmol} / 1(216 \mathrm{mg} /$ $100 \mathrm{ml}$ ).

Throughout the trial all were given a diet in which the polyunsaturated :saturated ratio was increased. This diet was started four months before randomisation to exclude men whose plasma concentrations of cholesterol and LDL cholesterol were particularly sensitive to diet. It actually achieved a rise in the polyunsaturated:saturated ratio from 0.48 to 0.67 over the seven years. In addition to the change in diet, the bile acid sequestrant cholestyramine was given at a dose of $24 \mathrm{~g} /$ day (six packets) to the active treatment group and a biologically inactive silica mixture was given (blind) to the control group. The average compliance in both groups was about four packets a day ( $23 \%$ took fewer than two daily), which was near to that predicted-and about all that could be expected in view of the tedium of having to take orange flavoured bulky powder with all main meals for seven years. No other drugs were givenindeed, intervention was restricted to diet and cholestyramine; no specific advice was given on smoking, exercise, or weight.

The lowering achieved in the plasma concentration of cholesterol was half $(-14 \%)$ that predicted $(-28 \%)$ and almost all occurred in LDL cholesterol. The change in plasma cholesterol in the control group was as predicted $(-4.9 \%)$, giving an overall decrement in the treated group of $8.5 \%$ for plasma cholesterol and $12 \cdot 6 \%$ for LDL cholesterol. There was a very small increase in high density lipoprotein (HDL) cholesterol, amounting after seven years to $2.8 \%$ compared with the control group, and this has been calculated to reduce the risk of coronary heart disease by about $3 \%$.

The amount of reduction in coronary heart disease $(-19 \%)$ was very close to that which would be expected from this degree of lowering of plasma concentrations of cholesterol and LDL cholesterol. ${ }^{3}$ In those with a $25 \%$ reduction in LDL cholesterol the decrease in coronary heart disease was more than half that of those at the pretreatment level. The degree of reduction in plasma lipids was directly related to the number of packets of cholestyramine taken daily. Both this and the control resin caused gastrointestinal side effects in many men: $68 \%$ of those receiving cholestyramine had such complaints after one year and $25 \%$ after seven years-heartburn, gas, belching, and bloating being common.

There was only a $7 \%$ reduction in overall mortality, with an increase in deaths due to accidents and violence, presumably due to chance. There were 21 incident and eight fatal cases of cancer in the gastrointestinal tract in the cholestyramine group and 11 incident and one fatal case in the control group. Curiously, six of the cancers were buccal or pharyngeal in the cholestyramine group compared with none in the placebo group; the occurrence of these rare cancers in six of 1906 men should not pass unnoticed, and a complete post-trial follow up is essential.

The results of the Lipid Research Clinics trial are consistent with those of the Oslo heart study, ${ }^{4}$ the World Health Organisation clofibrate trial, ${ }^{5}$ and the Los Angeles Veterans Administration dietary trial. ${ }^{6}$ The Oslo heart study comprised men with comparable very high plasma concentrations of cholesterol given a diet with a polyunsaturated :saturated ratio of nearly 1.0; and, though the trial was much smaller and compounded in interpretation because of concurrent reduction of cigarette smoking, there was a significant decrease $(-40 \%)$ in mortality from coronary heart disease. In the World Health Organisation clofibrate trial those in the top three deciles of cholesterol distribution were recruited and there was a $20 \%$ decrease in the incidence of coronary heart disease, though this was confined to non-fatal myocardial infarction $(-25 \%)$. The Veterans Administration trial of a diet with a polyunsaturated: saturated ratio of 1.5 was conducted in elderly men, where the serum cholesterol concentrations were less high, but again those with the highest values were the most benefited. Such 
analysis has not been presented for the Helsinki mental hospital study, though the results of this also suggested that reduction of raised plasma cholesterol by a high polyunsaturated:saturated ratio diet was associated with a reduction in incidence of coronary heart disease. ${ }^{7}$ The consistency in the results of these trials is impressive.

A further encouraging aspect of the Lipid Research Clinics trial is that regression of coronary atheroma has recently been reported, even though the resolution of the techniques used is still not perfected. ${ }^{8}$ Several studies are now being undertaken to determine the effects of diets and drugs on regression of atheromatous lesions in the femoral artery, as these are easier to study. If it can be shown that parallel changes take place in the femoral and coronary arteries, then it is probably reasonable to assume that when a beneficial effect is shown in femoral arterial lesions similar changes should occur in coronary lesions.

There are those who will be quick to extrapolate the positive findings of the Lipid Research Clinics trial to people with lower concentrations of serum cholesterol and LDL cholesterol, and many others who will wish to infer that cholesterol lowering diets will have a similar beneficial effect. But we should be wary against overinterpretation in either of these directions. The results of the trial apply only to the top 5\% at risk and, as the authors state, the trial was not designed to test the dietary hypothesis. So far, multiple risk factor intervention trials which have included cholesterol lowering diets have generally been unsuccessful, possibly because in these trials the amount by which the concentration of cholesterol was lowered was less and the motivation to observe a fairly stringent diet less. ${ }^{9}$ But the consistency in the results of all the primary prevention trials now suggests that at least those in the top quintile for plasma cholesterol should receive a diet with a high polyunsaturated:saturated ratio, perhaps with cholestyramine. The regimen must be sufficient to lower the plasma concentration of cholesterol over many years by about $10 \%$ and LDL cholesterol by $15 \%$. This requires a high degree of compliance; but if the need is explained adequately to people at high risk their motivation should be adequate.

There will also be others who will take encouragement and interpret the results in favour of the use of other cholesterol lowering drugs to prevent coronary heart disease. With the experience of the World Health Organisation clofibrate trial (which showed an increase in non-cardiovascular mortality) still fresh, however, the authors are surely right to state that "the mode of action, cholesterol lowering potency and possible toxicity of cholesterol lowering drugs must be taken into account before their use is advocated." The risk of correcting risk by drugs may be greater than the uncorrected risk. ${ }^{10}$

A far bigger effort is now needed to identify people with very high plasma cholesterol concentrations. There are three simple lines of approach which are more economic and practical methods of screening than that of taking blood from every man over 30 , particularly when only one in 20 will be affected. One is for hospital doctors to be assiduous in measuring plasma cholesterol (and, if high, LDL and HDL cholesterol) in all patients who develop angina or myocardial infarction under the age of 50 or 55 in order to identify high risk families; another is for cardiologists to liaise more closely with dermatologists, who not infrequently see patients with hypercholesterolaemic xanthomatosis; and a third is for family doctors to think hard about families where vascular disease has occurred prematurely or in excess and to screen all relatives for hypercholesterolaemia.

These trials of the effects of lowering cholesterol on coronary heart disease in high risk people have been tests of endurance for the participants, the architects, the investigators, and the analysts. Their perseverance has paid off, and patients with very considerable hypercholesterolaemia, particularly the young, should now be treated more vigorously. The fact that their poor prognosis may be changed is an important advance and should focus even more attention on the high risk strategy. ${ }^{9}$ But it should not be forgotten that the successful Lipid Research Clinics study was a trial using a gastrointestinal resin and not a diet trial and that it was limited to those in the top $5 \%$ of plasma cholesterol distribution.

Duke of Edinburgh Professor of Cardiology,

M F OLIVER

University of Edinburgh,

Cardiovascular Research Unit,

Edinburgh EH8 9XF

1 Anonymous. The Lipid Research Clinics coronary primary prevention trial results. I. Reduction in incidence of coronary heart disease. $\mathcal{F} A M A$ 1984;251 :351-64.

2 Anonymous. The coronary primary prevention trial: design and implementation. F Chronic Dis 1979;31:609-31.

${ }^{3}$ Anonymous. The Lipid Research Clinics coronary primary prevention trial results. II. The relationship of reduction in incidence of coronary heart disease to cholesterol lowering. fAMA 1984;251:365-74.

${ }^{4}$ Hjermann I, Byre KV, Holme I, Leren P. Effect of diet and smoking intervention on the incidence of coronary heart disease. Report from the Oslo study group of a randomized trial in healthy men. Lancet 1981 ;ii: 1303-10.

5 WHO Clofibrate Trial Committee of Principal Investigators. A co-operative trial in the primary prevention of ischaemic heart disease using clofibrate. Br Heart f 1978;40:1069-118.

${ }^{6}$ Dayton S, Pearce ML, Hashimoto S, et al. A controlled clinical trial of a diet high in unsaturated fat in preventing complications of atherosclerosis. Circulation 1969;40, suppl 2:1-63.

7 Turpeinen O, Karvonen MJ, Pekkarinen M, et al. Dietary prevention of coronary heart disease: the Finnish mental hospital study. Int $\mathcal{f}$ Epidemiol 1979;8:99-118.

${ }^{8}$ Levy RI. The influence of cholestyramine-induced lipid changes on coronary artery disease progression: the NHLBI type II coronary intervention study. (Abstract.) Arteriosclerosis 1983;3:481a.

9 Oliver MF. Should we not forget about mass control of coronary risk factors ? Lancet 1983;ii:37-8.

${ }^{10}$ Oliver MF. Risks of correcting the risks of coronary disease and stroke with drugs. $N$ Engl f Med $1982 ; 306: 297-8$.

\section{Smoking in pregnancy}

Most adults in Britain are now non-smokers, ${ }^{1}$ and this is true in all social classes. From 1972 to 1982 the proportion of men who were heavy smokers (20 or more cigarettes a day) decreased, but no such change has been seen in women who smoke heavily (who are mostly in social class V). The consequences of this differential flight from cigarettes by heavy smokers affects not only these women but their children.

The complex effects of smoking on pregnancy and its outcome have recently been examined by Sidle in a review of 336 papers on this topic. ${ }^{2}$ Concentrating on the relations between smoking and birth weight, perinatal mortality, and prematurity he has also examined the effects of smoking during pregnancy on handicap, pre-eclampsia, spontaneous abortion, and congenital abnormalities-as well as the different effects of carbon monoxide, cyanide, and new types of cigarette. The long term effects on the child after birth have been similarly reviewed.

As early as 1902 Ballantyne had found an increase in the abortion rate in French and Austrian women working in tobacco factories. In 1929 Knopf suggested that smoking itself might adversely affect the developing child, but interest in the subject was limited until the early 1970 s, when several studies showed an association between maternal smoking and reduced birth weight as a result of retardation of fetal intrauterine growth. ${ }^{3}$ Chronic fetal deficiency of oxygen was considered to be the result of 